\title{
CHERN-SIMONS REDUCTION AND NON-ABELIAN FLUID MECHANICS*
}

\author{
R. Jackiw and V.P. Nair ${ }^{\dagger}$ \\ Center for Theoretical Physics \\ Massachusetts Institute of Technology \\ Cambridge, MA 02139-430\%, USA \\ So-Young Pi \\ Physics Department \\ Boston University \\ Boston, MA 02215, USA
}

\begin{abstract}
We propose a non-Abelian generalization of the Clebsch parameterization for a vector in three dimensions. The construction is based on a group-theoretical reduction of the Chern-Simons form on a symmetric space. The formalism is then used to give a canonical (symplectic) discussion of non-Abelian fluid mechanics, analogous to the way the Abelian Clebsch parameterization allows a canonical description of conventional fluid mechanics.
\end{abstract}

\section{INTRODUCTION}

In a recent paper [1], a pure $S U(2)$ gauge potential $A=g^{-1} \mathrm{~d} g$, whose Chern-Simons term

$$
\begin{aligned}
C S(A) & =\int \omega(A) \\
\omega(A) & =-\frac{1}{8 \pi^{2}} \operatorname{tr}\left(A \mathrm{~d} A+\frac{2}{3} A^{3}\right)=\frac{1}{16 \pi^{2}}\left(A^{a} \mathrm{~d} A^{a}+\frac{1}{3} \epsilon^{a b c} A^{a} A^{b} A^{c}\right)
\end{aligned}
$$

measures the quantized winding number of $g$ [2],

$$
C S\left(g^{-1} \mathrm{~d} g\right)=\frac{1}{24 \pi^{2}} \int \operatorname{tr}\left(g^{-1} \mathrm{~d} g\right)^{3} \equiv W(g)
$$

was used to generate a $U(1)$ potential $a$, by projection onto an Abelian direction.

$$
a=-2 \operatorname{tr} t^{3} g^{-1} \mathrm{~d} g
$$

\footnotetext{
${ }^{*}$ This work is supported in part by funds provided by the U.S. Department of Energy (D.O.E.) under contract DE-FC02-94ER40818, DE-FG02-91ER40676 and by NSF grant PHY-9605216. MIT-CTP-2971, BU HEP-00-06, hep-th/0004084, April 2000

†Permanent address: Physics Department, City College of the CUNY, New York, NY 10031.
} 
$\left[t^{a}\right.$ are anti-Hermitian generators of the $S U(2)$ group, normalized by $\operatorname{tr} t^{a} t^{b}=-\frac{1}{2} \delta^{a b}$.] The Abelian potential $a$ is not a pure gauge, and it contains three arbitrary functions [corresponding to the three parameters of $S U(2)$ ], hence $a$ can represent an arbitrary Abelian 3-vector. The Chern-Simons 3-form for a coincides with that of its $S U(2)$ pure gauge antecedent, by virtue of the $S U(2)$ identity $\operatorname{tr}\left(t^{3} g^{-1} \mathrm{~d} g\right) \mathrm{d} \operatorname{tr}\left(t^{3} g^{-1} \mathrm{~d} g\right)=(1 / 3 !) \operatorname{tr}\left(g^{-1} \mathrm{~d} g\right)^{3}$. Thus the constructed Abelian potential possesses quantized Chern-Simons number (or magnetic helicity) [3] $\left(1 / 16 \pi^{2}\right) \int a \mathrm{~d} a$, equal to $W(g)$, the winding number of $g$. Because within $S U(2)$, $\operatorname{tr}\left(g^{-1} \mathrm{~d} g\right)^{3}$ can be explicitly presented as a total divergence [4], the Abelian Chern-Simons density $a \mathrm{~d} a$ also appears as a total divergence. This in turn indicates that the Clebsch parameterization for $a$

$$
a=\mathrm{d} \theta+\alpha \mathrm{d} \beta
$$

can be readily constructed. This parameterization of an Abelian potential $a$ ensures that the corresponding Chern-Simons density $a \mathrm{~d} a$ is a total divergence.

$$
\begin{aligned}
a \mathrm{~d} a=\mathrm{d} \theta \mathrm{d} \alpha \mathrm{d} \beta & =\mathrm{d}(\theta \mathrm{d} \alpha \mathrm{d} \beta) \\
& =-\mathrm{d}(\mathrm{d} \theta \alpha \mathrm{d} \beta) \\
& =\mathrm{d}(\mathrm{d} \theta \mathrm{d} \alpha \beta)
\end{aligned}
$$

In this paper we discuss how the above structures extend to the non-Abelian situation. Also we use our non-Abelian quantities to construct a canonical theory of non-Abelian fluid mechanics, analogous to the way in which the Abelian Clebsch parameterization is used in ordinary fluid mechanics.

We begin, in section II, with a pure gauge $g^{-1} \mathrm{~d} g$ in some non-Abelian group $G$ (called the Ur-group), and the Chern-Simons term again coincides with the winding number of $g$ as in (1.1), (1.2). We consider a normal subgroup $H$, with generators $I^{\alpha}$, and construct a non-Abelian gauge field by projection.

$$
A^{\alpha} \propto \operatorname{tr}\left(I^{\alpha} g^{-1} \mathrm{~d} g\right)
$$

Within $H$, this is not a pure gauge. We determine the group structure that is needed to ensure that the Chern-Simons 3-form $\omega(A)$ of $A^{\alpha}$ is proportional to $\operatorname{tr}\left(g^{-1} \mathrm{~d} g\right)^{3}$, so that the Chern-Simons number of $A^{\alpha}$ equals the winding number of $g$. In this way we construct non-Abelian gauge fields, belonging to the group $H$, with quantized Chern-Simons number. Moreover, we describe the properties of the Ur-group $G$, that are needed so that the projected potential $A^{\alpha}$ enjoys sufficient generality to represent an arbitrary potential in $H$.

Since $\operatorname{tr}\left(g^{-1} \mathrm{~d} g\right)^{3}$ is a total derivative for an arbitrary group (although this fact cannot in general be expressed in finite terms) [5] our construction ensures that the form of $A^{\alpha}$, which is achieved through the projection (1.6), produces a total derivative expression for its Chern-Simons density $\omega(A)$.

With the above mentioned properties for the potential, it is appropriate to consider (1.6) as a "non-Abelian Clebsch parameterization".

In explicit examples, which we present in section III, it is found that the "total derivative" form for the Chern-Simons density of $A^{\alpha}$ is achieved in two steps. The parameterization (1.6) directly leads to an Abelian form of the Chern-Simons density

$$
A^{\alpha} \mathrm{d} A^{\alpha}+\frac{1}{3} f^{\alpha \beta \gamma} A^{\alpha} A^{\beta} A^{\gamma}=\gamma \mathrm{d} \gamma
$$

for some $\gamma$. Then Darboux's theorem [6] (or usual fluid dynamical theory [7]) ensures that $\gamma$ can be presented in Clebsch form, so that $\gamma \mathrm{d} \gamma$, is explicitly a total derivative. 
We also observe that at least for $S U(2)$ one can do without the above general discussion and directly present a parameterization for arbitrary $S U(2)$ potentials, which produces a total derivative expression for the $S U(2)$ Chern-Simons density. The parameterization is a natural generalization into the non-Abelian context of the Clebsch parameterization (1.4), which achieves the total derivative form for Abelian Chern-Simons densities.

In section IV, we construct a non-Abelian version of fluid mechanics and magnetohydrodynamics, which may be useful as an effective description for the long wavelength degrees of freedom in a quark-gluon plasma.

\section{PARAMETERIZATION OF POTENTIALS AND THE CHERN-SIMONS 3-FORM}

\section{A. General Considerations}

As stated in the introduction, we consider the parameterization of gauge potentials for a group $H$ of the form $\operatorname{tr}\left(I^{\alpha} g^{-1} \mathrm{~d} g\right)$, where $g$ is an element of a group $G, H$ being a subgroup of $G$ and $I^{\alpha}$ are the generators of $H$. Conditions on the Ur-group $G$, which we take to be compact and semi-simple, are the following. First of all $G$ has to be so chosen that it has sufficient number of parameters to make $\operatorname{tr}\left(I^{\alpha} g^{-1} \mathrm{~d} g\right)$ a generic potential for $H$. Since we are in three dimensions, an $H$-potential $A_{i}^{\alpha}$ has $3 \times \operatorname{dim} H$ independent functions; so a minimal requirement will be

$$
\operatorname{dim} G \geq 3 \operatorname{dim} H
$$

Secondly we require that the $H$-Chern-Simons form for $A^{\alpha}$ should coincide with that of $g^{-1} \mathrm{~d} g$, thereby ensuring that the $H$-potential possesses (quantized) Chern-Simons number equal to $W(g)$ and also that the $H$-Chern-Simons density is a total derivative, or at least can be brought to an Abelian form as in (1.7). As we shall show in a moment, this is achieved if $G / H$ is a symmetric space. In this case, if we split the Lie algebra of $G$ into the $H$-subalgebra spanned by $I^{\alpha}, \alpha=1, \ldots, \operatorname{dim} H$, and the orthogonal complement spanned by $S^{A}, A=1, \ldots,(\operatorname{dim} G-\operatorname{dim} H)$, the commutation rules are of the form

$$
\begin{aligned}
{\left[I^{\alpha}, I^{\beta}\right] } & =f^{\alpha \beta \gamma} I^{\gamma} \\
{\left[I^{\alpha}, S^{A}\right] } & =h^{\alpha A B} S^{B} \\
{\left[S^{A}, S^{B}\right] } & =c h^{\alpha A B} I^{\alpha}
\end{aligned}
$$

$\left(h^{\alpha}\right)^{A B}$ form a (possibly reducible) representation of the $H$-generators $I^{\alpha}$. The constant $c$ depends on normalizations. More explicitly, if the structure constants for the Ur-group $G$ are named $\bar{f}^{a b c}, a, b, c=1, \ldots, \operatorname{dim} G$, then the conditions $2.2 \mathrm{a}-\mathrm{c}$ ) require that $\bar{f}^{a b c}$ vanishes whenever an odd number of indices belongs to the orthogonal complement labeled by $A, B, \ldots$ Moreover, $f^{\alpha \beta \gamma}$ are taken to be the conventional structure constants for $H$ and this may render them proportional to (rather than equal to) $\bar{f}^{\alpha \beta \gamma}$.

We define the traces of the generators by

$$
\begin{aligned}
\operatorname{tr}\left(I^{\alpha} I^{\beta}\right) & =-a \delta^{\alpha \beta}, \quad \operatorname{tr}\left(S^{A} S^{B}\right)=-b \delta^{A B} \\
\operatorname{tr}\left(I^{\alpha} S^{A}\right) & =0 .
\end{aligned}
$$

We can evaluate the quantity $\operatorname{tr}\left[S^{A}, S^{B}\right] I^{\alpha}=\operatorname{tr} S^{A}\left[S^{B}, I^{\alpha}\right]$ using the commutation rules and (2.3). This immediately gives the relation $a c=b$. 
Expanding $g^{-1} \mathrm{~d} g$ in terms of generators, we write

$$
g^{-1} \mathrm{~d} g=\left(I^{\alpha} A^{\alpha}+S^{A} \alpha^{A}\right)
$$

which defines the $H$-potential $A^{\alpha}$. Equivalently

$$
A^{\alpha}=-\frac{1}{a} \operatorname{tr}\left(I^{\alpha} g^{-1} \mathrm{~d} g\right)
$$

(Such projected potentials have been used before in formulating sigma models, see [8]). From $\mathrm{d}\left(g^{-1} \mathrm{~d} g\right)=-g^{-1} \mathrm{~d} g g^{-1} \mathrm{~d} g$, we get the Maurer-Cartan relations

$$
\begin{aligned}
F^{\alpha} \equiv \mathrm{d} A^{\alpha}+\frac{1}{2} f^{\alpha \beta \gamma} A^{\beta} A^{\gamma} & =-\frac{c}{2} h^{\alpha A B} \alpha^{A} \alpha^{B} \\
\mathrm{~d} \alpha^{A}+h^{\alpha B A} A^{\alpha} \alpha^{B} & =0 .
\end{aligned}
$$

Using these results, the following chain of equations shows that the Chern-Simons 3-form for the $H$-gauge group is proportional to $\operatorname{tr}\left(g^{-1} \mathrm{~d} g\right)^{3}$.

$$
\begin{aligned}
\omega(A) & =\frac{1}{16 \pi^{2}}\left(A^{\alpha} \mathrm{d} A^{\alpha}+\frac{1}{3} f^{\alpha \beta \gamma} A^{\alpha} A^{\beta} A^{\gamma}\right) \\
& =\frac{1}{48 \pi^{2}}\left(A^{\alpha} \mathrm{d} A^{\alpha}+2 A^{\alpha} F^{\alpha}\right) \\
& =\frac{1}{48 \pi^{2}}\left(A^{\alpha} \mathrm{d} A^{\alpha}+c h^{\alpha A B} A^{\alpha} \alpha^{A} \alpha^{B}\right) \\
& =\frac{1}{48 \pi^{2}}\left(A^{\alpha} \mathrm{d} A^{\alpha}+c \mathrm{~d} \alpha^{A} \alpha^{A}\right) \\
& =-\frac{1}{48 \pi^{2}}\left[\frac{1}{a} \operatorname{tr}(A \mathrm{~d} A)+\frac{c}{b} \operatorname{tr}(\mathrm{d} \alpha \alpha)\right] \\
& =-\frac{1}{48 \pi^{2} a} \operatorname{tr}(A \mathrm{~d} A+\alpha \mathrm{d} \alpha) \\
& =-\frac{1}{48 \pi^{2} a} \operatorname{tr} g^{-1} \mathrm{~d} g \mathrm{~d}\left(g^{-1} \mathrm{~d} g\right) \\
& =\frac{1}{48 \pi^{2} a} \operatorname{tr}\left(g^{-1} \mathrm{~d} g\right)^{3}
\end{aligned}
$$

In the above sequence of manipulations, we have used the Maurer-Cartan relations (2.6), which rely on the symmetric space structure of (2.2a-c), and the trace relations (2.3), along with $a c=b$.

We thus see that $\int \omega(A)$ is indeed the winding number of the configuration $g \in G$. Since $\operatorname{tr}\left(g^{-1} \mathrm{~d} g\right)^{3}$ is a total derivative locally on $G$, the potential (2.5), with the symmetric space structure of (2.2a-c), does indeed obey the requirement of making $\omega(A)$ a total derivative. It is therefore appropriate to call our construction (2.5) a "non-Abelian Clebsch parameterization".

\section{B. Choosing the Ur-group $G$}

In explicit realizations, given a gauge group of interest $H$, we need to choose a group $G$ such that the conditions (2.1), (2.2a-c) hold. In general this is not possible. However, one

can proceed recursively. Let us suppose that the desired result has been established for a group, which we call $H_{2}$. Then we form $H \subset G$ obeying (2.2a-c) as $H=H_{1} \times H_{2}$, where $H_{1}$ 
is the gauge group of interest, satisfying $\operatorname{dim} G \geq 3 \operatorname{dim} H_{1}$. For this choice of $H$, the result (IIA) becomes

$$
\omega\left(H_{1}\right)+\omega\left(H_{2}\right)=\frac{1}{48 \pi^{2} a} \operatorname{tr}\left(g^{-1} \mathrm{~d} g\right)^{3}
$$

But since $\omega\left(H_{2}\right)$ is already known to be a total derivative, (2.8) shows the desired result: $\omega\left(H_{1}\right)$ is a total derivative.

As a specific example, consider the orthogonal groups $O(n)$ for which we can use $G=$ $O(2 n-1)$ and $H=O(n) \times O(n-1)$. The case $n=2$, with $O(1)=\mathbf{1}$, reproduces the previous results of the Abelian construction $O(3)$ [or $S U(2)] \rightarrow O(2)$ [四]. For $n=3, G$ is $O(5), H_{1}=O(3)$ and $H_{2}=O(2)$. Since $\omega[O(2)]$ is already known to be a total derivative, we learn from (2.8) that the Chern-Simons density for $O(3)[S U(2)]$ is also a total derivative. (Explicit formulas for this case are presented in the next section.) Evidently the procedure can be continued for arbitrary $O(n)$, but we have not found a simple sequence of embeddings for other groups.

To see that the algebra of $G=O(2 n-1)$ and $H=O(n) \times O(n-1)$ satisfies (2.2a-c) we proceed as follows. Let $\Gamma^{\alpha}$ denote the set of Dirac gamma matrices in $n$ dimensions and let $\gamma^{i}$ denote the set of Dirac gamma matrices in $(n-1)$ dimensions. These are considered as acting on different vector spaces. We have

$$
\begin{array}{r}
\Gamma^{\alpha} \Gamma^{\beta}+\Gamma^{\beta} \Gamma^{\alpha}=2 \delta^{\alpha \beta} \\
\gamma^{i} \gamma^{j}+\gamma^{j} \gamma^{i}=2 \delta^{i j}
\end{array}
$$

We construct

$$
\begin{aligned}
\Sigma^{\alpha \beta} & =\frac{i}{2}\left[\Gamma^{\alpha}, \Gamma^{\beta}\right] \\
\sigma^{i j} & =\frac{i}{2}\left[\gamma^{i}, \gamma^{j}\right] \\
S^{\alpha i} & =\Gamma^{\alpha} \gamma^{i}
\end{aligned}
$$

$\Sigma^{\alpha \beta}$ are (Hermitian) generators of $O(n), \sigma^{i j}$ are generators of $O(n-1)$. The set of matrices $\left(\Sigma^{\alpha \beta}, \sigma^{i j}, S^{\alpha i}\right)$ form the generators of $O(2 n-1)$. From (2.10) we find that $S^{\alpha i}$ is a vector of $O(n)$ and a vector of $O(n-1)$ and also that

$$
i\left[S^{\alpha i}, S^{\beta j}\right]=2 \delta^{i j} \Sigma^{\alpha \beta}+2 \delta^{\alpha \beta} \sigma^{i j}
$$

We have thus the required structure $(2.2 \mathrm{a}-\mathrm{c})$. We construct the $O(n)$-gauge potential as

$$
A^{\alpha \beta}=\frac{i}{a} \operatorname{tr}\left(\Sigma^{\alpha \beta} g^{-1} \mathrm{~d} g\right)
$$

The number of arbitrary functions present in $A^{\alpha \beta}$ defined by (2.12) is $\frac{3}{2} n(n-1)$, which is exactly the right number for an $O(n)$-gauge potential in three dimensions, so that (2.1) is satisfied in just the right way (as an equality). This result is seen as follows. The $O(2 n-1)$ group element $g$ depends on $(n-1)(2 n-1)$ parameters. However the trace with $\Sigma^{\alpha \beta}$ removes dependence on the $\frac{1}{2}(n-1)(n-2)$ parameters of the $O(n-1)$ subgroup. This is a consequence of the fact that $A^{\alpha \beta}$ is unchanged when $g$ is replaced by $g k$, with $k \in O(n-1)$.

$$
\begin{aligned}
\operatorname{tr}\left(\sum^{\alpha \beta} g^{-1} \mathrm{~d} g\right) & \rightarrow \operatorname{tr}\left[\sum^{\alpha \beta}(g k)^{-1} \mathrm{~d}(g k)\right] \\
& =\operatorname{tr}\left[\sum^{\alpha \beta}\left(k^{-1}\left(g^{-1} \mathrm{~d} g\right) k+k^{-1} \mathrm{~d} k\right)\right] \\
& =\operatorname{tr}\left(k \Sigma^{\alpha \beta} k^{-1} g^{-1} \mathrm{~d} g\right)+\operatorname{tr}\left(\Sigma^{\alpha \beta} k^{-1} \mathrm{~d} k\right)
\end{aligned}
$$


The second term on the right vanishes due to the orthogonality of traces of $O(n)$ with $O(n-1)$ generators, while $k$ disappears from the first since it commutes with $\Sigma^{\alpha \beta}$.

In the above construction, one can also define an $O(n-1)$-potential

$$
A^{i j}=\frac{i}{a} \operatorname{tr}\left(\sigma^{i j} g^{-1} \mathrm{~d} g\right)
$$

Indeed, this is the potential that enters $\omega\left(H_{2}\right)=\omega[O(n-1)]$. This potential depends on the functions used to construct $A^{\alpha \beta}$ in (2.12). Thus (2.14) does not give an independent $O(n-1)$ potential. But this is immaterial since we are really interested in the $O(n)$-potential; (2.14) enters our discussion only in the formula for the Chern-Simons density, namely $\omega[O(n)]=$ $\left(1 / 48 \pi^{2} a\right) \operatorname{tr}\left(g^{-1} \mathrm{~d} g\right)^{3}-\omega[O(n-1)]$.

As we have already noted, the potential (2.12) depends on just the right number of arbitrary functions. We shall now show explicitly that it is sufficiently general to reproduce an arbitrary $O(n)$-gauge potential that lies close to the trivial gauge orbit $A^{\alpha}=0$ (or $A^{\alpha}=$ pure gauge). [For compactness we rename $A^{\alpha \beta}$ of (2.12) simply as $A^{\alpha}$.] Potentials in the neighborhood of the trivial gauge orbit may be obtained by writing $g=\exp \left(i S_{A} \theta^{A}\right) \cdot h k$, $h \in H_{1}=O(n), k \in H_{2}=O(n-1)$. Expanding in powers of $\theta^{A}$, we then find

$$
\begin{aligned}
A^{\alpha} & =R^{\alpha \gamma}(h) a^{\gamma}+\left(h^{-1} \mathrm{~d} h\right)^{\alpha} \\
a^{\gamma} & \approx \frac{c}{2} h^{\gamma A B} \theta^{B} \mathrm{~d} \theta^{A}+\cdots
\end{aligned}
$$

where $R^{\alpha \gamma}(h)$ is defined by

$$
h I^{\alpha} h^{-1}=R^{\alpha \gamma}(h) I^{\gamma}
$$

( $k \in H_{2}$ drops out of the expression for the potential as discussed earlier.) Eq. (2.15) tells us that $A^{\alpha}$ is the gauge transform of the potential $a^{\gamma}$. For small $\theta^{\prime}$ s, this can be brought to the Clebsch form for each value of the Lie algebra index $\gamma$. We can see this as follows. There are $n(n-1)=2 \operatorname{dim} H_{1}$ functions $\theta^{A}$ in the expression for $a^{\gamma}$. [Additional $\frac{1}{2} n(n-1)$ parameters are contained in $h$, giving the total of $\frac{3}{2} n(n-1)$ parameters for the potential (2.12).] $h^{\gamma A B}$ is antisymmetric in $A, B$. By choosing an appropriate basis one can present the commutator (2.20) in the form $\left[S^{(\gamma)}, \tilde{S}^{(\gamma)}\right] \propto I^{\gamma}, \gamma=1,2, \ldots, \operatorname{dim} H_{1}=\frac{1}{2} n(n-1)$. (There is no summation over $\gamma \cdot S^{(\gamma)}, \tilde{S}^{(\gamma)}$ are selected linear combinations of the $S^{A}$ 's.) In this basis, for each $\gamma$, $a^{\gamma} \approx \alpha^{(\gamma)} \mathrm{d} \beta^{(\gamma)}$, (no summation over $\gamma$ ), where $\alpha^{(\gamma)}$ and $\beta^{(\gamma)}$ are independent combinations of the $\theta^{A}$ 's. This manifestly display $\mathrm{s} a^{\gamma}$ in the Clebsch form for each value of $\gamma$. Since we know that any vector in three dimensions can be brought to the Clebsch form, Eq. (2.15) tells us that any gauge potential, which is sufficiently close to the trivial one, can be brought to the form (2.5). In other words, (2.5) [or (2.12)] is a general parameterization for gauge potentials in a small neighborhood of $A=0$ (or pure gauge) in the space $\mathcal{A}$ of three-dimensional gauge potentials. Since $\mathcal{A}$ is an affine space, it may be possible to extend this result over a larger neighborhood. A different way of stating this result is as follows. The arbitrary functions appearing in the expression for the potential, namely, the gauge parameters contained in $h$ and the coset parameters $\theta^{A}$, give a choice of coordinates on $\mathcal{A}$. This choice of coordinates is valid near the trivial gauge orbit or near the origin in the gauge-invariant configuration space $\mathcal{C}=\mathcal{A} / \mathcal{G}$, the space of gauge potentials modulo gauge transformations.

It is a well-known theorem, in the context of universal connections, that any gauge potential can be written in the form (2.5) for a sufficiently large group $G$ [9]. In general, this requires $\operatorname{dim} G \geq(d+1)(2 d+1)(\operatorname{dim} H)^{3}$ for gauge potentials of unitary groups in d dimensions. (The case of orthogonal groups can be realized as a special case of the unitary one, 
and a similar condition on the dimensions holds.) It is interesting to note that we have a parameterization of the gauge potential with the minimal number of parameters, namely, $3 \operatorname{dim} H$, which is significantly smaller than what appears in the construction of universal connections. It may be that our parameterization does not capture all the topological subtleties that gauge fields in three dimensions can have. It should also be pointed out that any parameterization, and not just ours, has drawbacks. This is because the configuration space $\mathcal{C}$, for non-Abelian groups, has nontrivial topology and hence one cannot choose coordinates globally valid on $\mathcal{C}$. (In the Abelian case, $\mathcal{C}$ is topologically trivial for fields on $\mathbf{R}^{3}$ and globally valid parameterizations exist.)

\section{THE $O(3)$ GAUGE POTENTIAL}

We take $G=O(5), H=O(3) \times O(2)$. We consider the 4-dimensional spinorial representation of $O(5)$. With the generators normalized by $\operatorname{tr}\left(t^{a} t^{b}\right)=-\delta^{a b}$, the Lie algebra generators of $O(5)$ are given by

$$
\begin{aligned}
I^{\alpha} & =\frac{1}{2 i}\left(\begin{array}{cc}
\sigma^{\alpha} & 0 \\
0 & \sigma^{\alpha}
\end{array}\right) \\
I^{0} & =\frac{1}{2 i}\left(\begin{array}{cc}
-1 & 0 \\
0 & 1
\end{array}\right) \\
S^{A} & =\frac{1}{i \sqrt{2}}\left(\begin{array}{cc}
0 & 0 \\
\sigma^{A} & 0
\end{array}\right) \quad \tilde{S}^{A}=\frac{1}{i \sqrt{2}}\left(\begin{array}{cc}
0 & \sigma^{A} \\
0 & 0
\end{array}\right)
\end{aligned}
$$

$\sigma$ 's are the $2 \times 2$ Pauli matrices. $I^{\alpha}$ generate $O(3)$, with the conventional structure constants $\epsilon^{\alpha \beta \gamma}$, and $I^{0}$ is the generator of $O(2)$. S, $\tilde{S}$ are the coset generators.

A general group element in $O(5)$ can be written in the form $g=M h k$ where $h \in O(3)$, $k \in O(2)$, and

$$
M=\frac{1}{\sqrt{1+\overline{\mathbf{w}} \cdot \mathbf{w}-\frac{1}{4}(\mathbf{w} \times \overline{\mathbf{w}})^{2}}}\left(\begin{array}{cc}
1-\frac{i}{2}(\mathbf{w} \times \overline{\mathbf{w}}) \cdot \sigma & -\mathbf{w} \cdot \sigma \\
\overline{\mathbf{w}} \cdot \sigma & 1+\frac{i}{2}(\mathbf{w} \times \overline{\mathbf{w}}) \cdot \sigma
\end{array}\right)
$$

$w^{\alpha}$ is a complex 3-dimensional vector, with the bar denoting complex conjugation. $\mathbf{w} \cdot \overline{\mathbf{w}}=$ $w^{\alpha} \bar{w}^{\alpha}$ and $(\mathbf{w} \times \overline{\mathbf{w}})^{\alpha}=\epsilon^{\alpha \beta \gamma} w^{\beta} \bar{w}^{\gamma}$. The gauge potential given by $-\operatorname{tr}\left(I^{\alpha} g^{-1} \mathrm{~d} g\right)$ reads

$$
\begin{array}{r}
A^{\alpha}=R^{\alpha \beta}(h) a^{\beta}+\left(h^{-1} \mathrm{~d} h\right)^{\alpha} \\
a^{\alpha}=\frac{1}{1+\mathbf{w} \cdot \overline{\mathbf{w}}-\frac{1}{4}(\mathbf{w} \times \overline{\mathbf{w}})^{2}}\left\{\frac{w^{\alpha} \mathrm{d} \overline{\mathbf{w}} \cdot(\mathbf{w} \times \overline{\mathbf{w}})+\bar{w}^{\alpha} \mathrm{d} \mathbf{w} \cdot(\overline{\mathbf{w}} \times \mathbf{w})}{2}\right. \\
\left.+\epsilon^{\alpha \beta \gamma}\left(\mathrm{d} w^{\beta} \bar{w}^{\gamma}-w^{\beta} \mathrm{d} \bar{w}^{\gamma}\right)\right\}
\end{array}
$$

$A^{\alpha}$ is the $h$-gauge transform of $a^{\alpha}$ which depends on six parameters $\left(w^{\alpha}, \bar{w}^{\alpha}\right)$. The three gauge parameters of $h \in O(3)$, along with the six $\left(w^{\alpha}, \bar{w}^{\alpha}\right)$, give the nine functions needed to parameterize a general $O(3)$ - [or $S U(2)$-] potential in three dimensions. The Chern-Simons form is

$$
\begin{aligned}
\omega(A)= & \frac{1}{16 \pi^{2}}\left(A^{\alpha} \mathrm{d} A^{\alpha}+\frac{1}{3} \epsilon^{\alpha \beta \gamma} A^{\alpha} A^{\beta} A^{\gamma}\right) \\
= & \left.\frac{1}{16 \pi^{2}}\left(a^{\alpha} \mathrm{d} a^{\alpha}+\frac{1}{3} \epsilon^{\alpha \beta \gamma} a^{\alpha} a^{\beta} a^{\gamma}\right)-\mathrm{d}\left[\frac{1}{16 \pi^{2}}\left(\mathrm{~d} h h^{-1}\right)^{\alpha} a^{\alpha}\right)\right] \\
& \quad+\frac{1}{24 \pi^{2}} \operatorname{tr}\left(h^{-1} \mathrm{~d} h\right)^{3}
\end{aligned}
$$


The second equality reflects the usual response of the Chern-Simons density to gauge transformations. Using the explicit form of $a^{\alpha}$ as given in (3.3), we can further reduce this. Indeed we find that

$$
\begin{gathered}
a^{\alpha} \mathrm{d} a^{\alpha}+\frac{1}{3} \epsilon^{\alpha \beta \gamma} a^{\alpha} a^{\beta} a^{\gamma}=(-2) \frac{(\overline{\mathbf{w}} \times \mathrm{d} \overline{\mathbf{w}}) \cdot \rho+(\mathbf{w} \times \mathrm{d} \mathbf{w}) \cdot \bar{\rho}}{\left[1+\mathbf{w} \cdot \overline{\mathbf{w}}-\frac{1}{4}(\mathbf{w} \times \overline{\mathbf{w}})^{2}\right]^{2}} \\
\rho_{k}=\frac{1}{2} \epsilon_{i j k} \mathrm{~d} \bar{w}^{i} \mathrm{~d} \bar{w}^{j}
\end{gathered}
$$

Defining an Abelian potential

$$
a=\frac{\mathbf{w} \cdot \mathrm{d} \overline{\mathbf{w}}-\overline{\mathbf{w}} \cdot \mathrm{d} \mathbf{w}}{1+\mathbf{w} \cdot \overline{\mathbf{w}}-\frac{1}{4}(\mathbf{w} \times \overline{\mathbf{w}})^{2}}
$$

we can easily check that $a \mathrm{~d} a$ reproduces (3.5). In other words

$$
\omega(A)=\frac{1}{16 \pi^{2}} a \mathrm{~d} a+\mathrm{d}\left[\frac{\left.\left(\mathrm{d} h h^{-1}\right)^{\alpha} a^{\alpha}\right)}{16 \pi^{2}}\right]+\frac{1}{48 \pi^{2}} \operatorname{tr}\left(h^{-1} \mathrm{~d} h\right)^{3}
$$

If desired, the Abelian potential $a$ can now be written in the Clebsch form making $a \mathrm{~d} a$ into a total derivative.

The $O(3)$-potential (3.3) can also be written in a more compact form as

$$
a^{\alpha}=\frac{2 i}{\left(1+2 \bar{\xi} \cdot \xi+N^{2}\right)}\left\{\mathrm{d} \bar{\xi} J^{\alpha} \xi-\bar{\xi} J^{\alpha} \mathrm{d} \xi-N J^{\alpha} \mathrm{d} N\right\}
$$

where $N^{\alpha}=\bar{\xi} J^{\alpha} \xi, w_{\beta}=\sqrt{2} \xi_{\beta} .\left(J^{\alpha}\right)_{\beta \gamma}=-i \epsilon^{\alpha \beta \gamma}$ is the adjoint representation of the Lie algebra of $S U(2)$.

The Abelian gauge potential obtained in [1] by projection from $S U(2)$, in other words the potential (1.3), can also be written in a form very similar to the above expression for $a^{\alpha}$. With $g$ parameterized as

$$
g=\left(\begin{array}{cc}
\sqrt{1+(\bar{\xi} \xi)^{2}} & \sqrt{2} \xi \\
-\sqrt{2} \bar{\xi} & \sqrt{1+(\bar{\xi} \xi)^{2}}
\end{array}\right) \cdot e^{-i \sigma^{3} \theta / 2}
$$

the projection (1.3) gives

$$
a=\mathrm{d} \theta+\frac{2 i}{\left[1+2 \bar{\xi} \xi+(\bar{\xi} \xi)^{2}\right]}(\mathrm{d} \bar{\xi} \xi-\bar{\xi} \mathrm{d} \xi)
$$

where $\xi$ is now just a single complex function. [The Clebsch parameters $\alpha, \beta$ are given by $\xi=\sqrt{\rho} e^{i \beta}, \alpha=4 \rho /(1+\rho)^{2}$. ]

At least for the case of $O(3)$ [or $S U(2)]$, there is another way of parameterizing the potentials, without considering embeddings in a larger group. This also leads to the reduction of the Chern-Simons form as above. The key observation is the following. We can write

$$
\mathrm{d} \chi^{\alpha}=-\frac{1}{2} \epsilon^{\alpha \beta \gamma} \chi^{\beta} \chi^{\gamma}
$$

for $\chi^{\alpha}=i \operatorname{tr}\left(\sigma^{\alpha} g^{-1} \mathrm{~d} g\right), g \in S U(2)$. Further, $S U(2)$ being three-dimensional, we have

$$
\begin{aligned}
\chi^{1} \chi^{2} \chi^{3} & =\frac{1}{3 !} \epsilon^{\alpha \beta \gamma} \chi^{\alpha} \chi^{\beta} \chi^{\gamma} \\
& =-\chi^{1} \mathrm{~d} \chi^{1}=-\chi^{2} \mathrm{~d} \chi^{2}=-\chi^{3} \mathrm{~d} \chi^{3}
\end{aligned}
$$


We take each Lie algebra component of the potential to be proportional to $\chi^{\alpha}$,

$$
A^{\alpha}=f_{\alpha} \chi^{\alpha}
$$

with no summation over $\alpha$, i.e., Eq.(3.13) holds for each component separately. Using (3.12), (3.13) we then find

$$
\begin{aligned}
\omega(A) & =\frac{1}{16 \pi^{2}}\left(2 f_{1} f_{2} f_{3}-f_{1}^{2}-f_{2}^{2}-f_{3}^{2}\right) \chi^{1} \chi^{2} \chi^{3} \\
& =\frac{1}{16 \pi^{2}}\left(f^{2}-2 f_{1} f_{2} f_{3}\right) \chi^{3} \mathrm{~d} \chi^{3} \\
& =\frac{1}{16 \pi^{2}} a \mathrm{~d} a
\end{aligned}
$$

where $a=\sqrt{f^{2}-2 f_{1} f_{2} f_{3}} \chi^{3}$. We thus get the Abelian form again for a suitably defined Abelian potential $a$. $A^{\alpha}$ of (3.13) contains six parameters, three from $g$ and the three $f_{\alpha}$ 's. These, along with the three gauge parameters, [not displayed in (3.13)], give the requisite number of nine parameters. The Abelianization of the Chern-Simons form via $a$ works only in regions where $\sum f_{\alpha}^{2} \geq 2 f_{1} f_{2} f_{3}$, so that the square root is well defined. If this is not the case, one needs to use the absolute value of $\sum f_{\alpha}^{2}-2 f_{1} f_{2} f_{3}$ to get a real $a$. This can lead to some nonanalyticity in $a$ as a function of the spatial coordinates.

\section{TOWARDS A NON-ABELIAN FLUID MECHANICS}

We now turn to the question of whether our results can be used in a physical context. First of all, there has recently been renewed interest in general parameterizations of gauge fields, with the hope that the low energy physics of gauge theories might be clearer in certain cleverly chosen parameterizations [10]. Our work certainly fits in with this general philosophy.

Secondly, notice that the Clebsch parameterization and the consequent reduction of the Chern-Simons form are very useful in analyzing the evolution of magnetic helicity [1]. Considerations of a non-Abelian analogue of magnetic helicity, which may be relevant in the symmetry restored phase of the standard electroweak theory, for example, can be significantly aided by our analysis.

However, we now turn to a possible third application of our results: the construction of non-Abelian fluid mechanics that may be relevant to the analysis of collective modes in the quark-gluon plasma. The free Hamiltonian for nonrelativistic fluid mechanics is given by

$$
H=\int \mathrm{d}^{3} r \frac{1}{2} \rho \mathbf{v}^{2}
$$

where $\rho$ is the matter density field and $\mathbf{v}$ is the velocity field. The free evolution equations that these quantities satisfy are

$$
\begin{array}{r}
\frac{\partial \rho}{\partial t}+\nabla \cdot(\rho \mathbf{v})=0 \\
\frac{\partial \mathbf{v}}{\partial t}+(\mathbf{v} \cdot \nabla) \mathbf{v}=0
\end{array}
$$

The first is the continuity equation linking the current $\mathbf{j} \equiv \rho \mathbf{v}$ to the density; the second is the free Euler equation, stating that the acceleration vanishes. These equations can be obtained by Poisson bracketing with $H$, provided the nonvanishing brackets for $\rho, \mathbf{v}$ are 


$$
\begin{gathered}
\left\{v_{i}(\mathbf{r}), \rho\left(\mathbf{r}^{\prime}\right)\right\}=\frac{\partial}{\partial r^{i}} \delta\left(\mathbf{r}-\mathbf{r}^{\prime}\right) \\
\left\{v_{i}(\mathbf{r}), v_{j}\left(\mathbf{r}^{\prime}\right)\right\}=-\frac{\omega_{i j}(\mathbf{r})}{\rho(\mathbf{r})} \delta\left(\mathbf{r}-\mathbf{r}^{\prime}\right)
\end{gathered}
$$

where $\omega_{i j}=\partial_{i} v_{j}-\partial_{j} v_{i}$ is the vorticity [11. A natural question is whether there exists a canonical 1-form and a corresponding symplectic 2-form that give the Poisson bracket algebra (4.3a,b). The raison d'être for the Clebsch parameterization (for the vector field $\mathbf{v})$ is to provide this canonical formulation of fluid mechanics [12. One verifies that if $\mathbf{v}$ is presented as

$$
\mathbf{v}=\nabla \theta+\alpha \nabla \beta
$$

where the canonical pairs of variables are identified as $(\rho, \theta)$ and $(\rho \alpha, \beta)$, i.e.,

$$
\begin{aligned}
\left\{\theta(\mathbf{r}), \rho\left(\mathbf{r}^{\prime}\right)\right\} & =\delta\left(\mathbf{r}-\mathbf{r}^{\prime}\right) \\
\left\{\beta(\mathbf{r}), \rho \alpha\left(\mathbf{r}^{\prime}\right)\right\} & =\delta\left(\mathbf{r}-\mathbf{r}^{\prime}\right)
\end{aligned}
$$

then the algebra $4.3 \mathrm{a}, \mathrm{b})$ is reproduced.1

A Lagrangian that incorporates the canonical 1-form and the parameterization (4.4) is

$$
L=\int \mathrm{d}^{3} r\left[\frac{1}{2} \rho \mathbf{v}^{2}+\theta(\dot{\rho}+\nabla \cdot(\rho \mathbf{v}))-\rho \alpha(\dot{\beta}+\mathbf{v} \cdot \nabla \beta)\right]
$$

The time-derivative terms (denoted by the over-dot) supply the 1-form, while variation with respect to $\mathbf{v}$ yields (4.4). Apart from total derivatives, $L$ can also be presented as

$$
L=\int \mathrm{d}^{3} r\left[-\rho(\dot{\theta}+\alpha \dot{\beta})-\mathbf{j} \cdot(\nabla \theta+\alpha \nabla \beta)+\frac{\mathbf{j}^{2}}{2 \rho}\right]
$$

where we now use $\mathbf{j}$ instead of $\rho \mathbf{v}$ and the Clebsch variables have been clearly exposed. Since the construction (1.3) of an Abelian vector from an $S U(2)$ pure gauge presents that vector in Clebsch form, we may replace (4.7) by

$$
L=\int \mathrm{d}^{3} r\left[2 j^{\mu} \operatorname{tr}\left(t^{3} g^{-1} \partial_{\mu} g\right)-\sqrt{j^{\mu} j_{\mu}}\right]
$$

where now the kinetic term has also been generalized to the relativistic expression and $j^{\mu} \equiv(\rho, \mathbf{j})[13]$. In the nonrelativistic limit

$$
-\int \mathrm{d}^{3} r \sqrt{j^{\mu} j_{\mu}} \equiv-\int \mathrm{d}^{3} r \sqrt{\rho^{2}-\mathbf{j}^{2}} \longrightarrow-\int \mathrm{d}^{3} r \rho+\int \mathrm{d}^{3} r \frac{\mathbf{j}^{2}}{2 \rho}
$$

\footnotetext{
${ }^{1}$ The following observation allows understanding the need of the Clebsch parameterization for a canonical formulation. Although the algebra (4.3a, b) satisfies the Jacobi identity, it is nevertheless singular in that the Chern-Simons form constructed from the velocity

$$
C S(\mathbf{v})=\int \mathrm{d}^{3} r \epsilon^{i j k} v_{i} \partial_{j} v_{k}
$$

Poisson commutes with $\rho$ and $\mathbf{v}$; in other words, the Poisson algebra $4.3 \mathrm{a}, \mathrm{b})$ has a kernel given by the Chern-Simons form for the velocity. This is an obstruction to the construction of a symplectic 2 -form. The obstruction is removed when $\mathbf{v}$ is taken in the Clebsch parameterization, for then the Chern-Simons density is a total derivative and the Chern-Simons integral becomes a surface term with no bulk contribution.
} 
(The contribution $\int \mathrm{d}^{3} r \rho$ to $L$ is immaterial; it is a constant of motion.)

The formula (4.8) suggests a non-Abelian generalization. $j^{\mu}$ is promoted to an indexcarrying "color" current, $j^{\alpha \mu}$, and it is coupled to a non-Abelian, "Clebsch parameterized", vector constructed as in (2.5).

$$
L=\int \mathrm{d}^{3} r\left[-\frac{1}{a} j^{\alpha \mu} \operatorname{tr}\left(I^{\alpha} g^{-1} \partial_{\mu} g\right)-\sqrt{j^{\alpha \mu} j_{\mu}^{\alpha}}\right]
$$

Interaction with a dynamical gauge field can be included by promoting the derivative of $g$ to a gauge-covariant derivative, gauged on the right, i.e.,

$$
L=\int \mathrm{d}^{3} r\left[-\frac{1}{a} j^{\alpha \mu} \operatorname{tr}\left(I^{\alpha} g^{-1} D_{\mu} g\right)-\sqrt{j^{\alpha \mu} j_{\mu}^{\alpha}}\right]-\frac{1}{4} \int \mathrm{d}^{3} r F^{\alpha \mu \nu} F_{\mu \nu}^{\alpha}
$$

with

$$
D_{\mu} g=\partial_{\mu} g-e g A_{\mu}
$$

$A_{\mu}=A_{\mu}^{\alpha} I^{\alpha}$ are independent non-Abelian gauge potentials (not given by $g$ ) leading to the field strengths $F_{\mu \nu}^{\alpha}$. The gauge transformation properties are

$$
\begin{array}{ll}
g^{\prime} & =g h, \quad A^{\prime}=h^{-1} A h+\frac{1}{e} h^{-1} \mathrm{~d} h \\
j_{\mu}^{\prime} & =h^{-1} j_{\mu} h
\end{array}
$$

where $j_{\mu}=j_{\mu}^{\alpha} I^{\alpha}$.

We expect that the Lagrangian (4.11) will describe non-Abelian magnetohydrodynamics, namely the dynamics of a fluid with non-Abelian charge coupled to non-Abelian fields. The current density will be $\mathbf{j}^{\alpha}$ as given by its equation of motion. This gluon hydrodynamics can be useful for non-Abelian plasmas, such as the quark-gluon plasma. Details of (4.11) and possible applications are under further study. In a related investigation, conventional fluid mechanics is generalized so that it enjoys a supersymmetry [14].

\section{Acknowledgments}

Conversations with S. Deser and A. Polychronakos initiated the research by one of us $(\mathrm{RJ})$, who also benefited from the suggestion by J. Mickelsson that a promising route to a non-Abelian Clebsch parameterization could be through group-theoretic reduction of the Chern-Simons term. V.P.N thanks I. Singer for useful comments. 


\section{REFERENCES}

[1] R. Jackiw and S.-Y. Pi, Phys. Rev. D (in press), hep-th/9911072.

[2] S. Deser, R. Jackiw and S. Templeton, Ann. Phys. (NY), 140, 372 (1982), (E) 185, 406 (1985).

[3] L. Woltier, Proc. Nat. Acad. Sci. 44, 489 (1958); H. Moffatt, J. Fluid Mech. 35, 117 (1969); M. Berger and G. Field, J. Fluid Mech. 147, 133 (1984).

[4] R. Jackiw in S. Treiman, R. Jackiw, B. Zumino and E. Witten, Current Algebra and Anomalies (Princeton University Press/World Scientific, Princeton, NJ/Singapore, 1985).

[5] C. Crönstrom and J. Mickelsson, J. Math. Phys. 24, 2528 (1983), (E) 27, 419 (1986).

[6] A constructive derivation of Darboux's theorem is given by R. Jackiw in Constraint Theory and Quantization Methods, F. Colomo, L. Lusanna and G. Marmo, eds. (World Scientific, Singapore, 1994), reprinted in R. Jackiw, Diverse Topics in Theoretical and Mathematical Physics (World Scientific, Singapore, 1995).

[7] H. Lamb, Hydrodynamics (Cambridge University Press, Cambridge, UK, 1932).

[8] A.P. Balachandran, A. Stern and C.G. Trahern, Phys. Rev. D19, 2416 (1979) and references therein.

[9] M.S. Narasimhan and S. Ramanan, Am. J. Math. 83, 563 (1961); ibid, 85, 223 (1963).

[10] L. Faddeev and A. Niemi, Phys. Lett. B464, 90 (1999); Phys. Rev. Lett. 82, 1624 (1999).

[11] P.J. Morrison and J.M. Greene, Phys. Rev. Lett. 45, 790 (1980); (E) 48, 569 (1982).

[12] C.C. Lin, in Proceedings of the International School of Physics "Enrico Fermi" (XXI), G. Careri, ed. (Academic Press, New York, NY, 1963).

[13] R. Jackiw and A. Polychronakos, Comm. Math. Phys. 207, 107 (1999).

[14] R. Jackiw and A. Polychronakos, to appear in Phys. Rev. D; hep-th/0004083. 